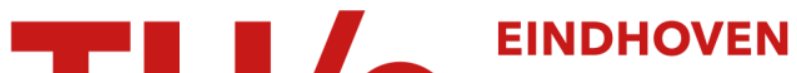 \\ UNIVERSITY OF \\ TECHNOLOGY
}

\section{Simulation of claylike colloids}

Citation for published version (APA):

Hecht, M., Harting, J. D. R., Ihle, T., \& Herrmann, H. J. (2005). Simulation of claylike colloids. Physical Review E - Statistical, Nonlinear, and Soft Matter Physics, 72(1), 011408-1/16. [011408].

https://doi.org/10.1103/PhysRevE.72.011408

DOI:

10.1103/PhysRevE.72.011408

Document status and date:

Published: 01/01/2005

\section{Document Version:}

Publisher's PDF, also known as Version of Record (includes final page, issue and volume numbers)

\section{Please check the document version of this publication:}

- A submitted manuscript is the version of the article upon submission and before peer-review. There can be important differences between the submitted version and the official published version of record. People interested in the research are advised to contact the author for the final version of the publication, or visit the $\mathrm{DOI}$ to the publisher's website.

- The final author version and the galley proof are versions of the publication after peer review.

- The final published version features the final layout of the paper including the volume, issue and page numbers.

Link to publication

\section{General rights}

Copyright and moral rights for the publications made accessible in the public portal are retained by the authors and/or other copyright owners and it is a condition of accessing publications that users recognise and abide by the legal requirements associated with these rights.

- Users may download and print one copy of any publication from the public portal for the purpose of private study or research.

- You may not further distribute the material or use it for any profit-making activity or commercial gain

- You may freely distribute the URL identifying the publication in the public portal.

If the publication is distributed under the terms of Article 25fa of the Dutch Copyright Act, indicated by the "Taverne" license above, please follow below link for the End User Agreement:

www.tue.nl/taverne

Take down policy

If you believe that this document breaches copyright please contact us at:

openaccess@tue.nl

providing details and we will investigate your claim. 


\title{
Simulation of claylike colloids
}

\author{
Martin Hecht, ${ }^{1}$ Jens Harting, ${ }^{1}$ Thomas Ihle, ${ }^{1,2}$ and Hans J. Herrmann ${ }^{1}$ \\ ${ }^{1}$ ICP, University of Stuttgart, Pfaffenwaldring 27, 70569 Stuttgart, Germany \\ ${ }^{2}$ North Dakota State University, Department of Physics, Box 5566, Fargo, North Dakota 58105-5566, USA
}

(Received 1 February 2005; published 26 July 2005)

\begin{abstract}
We investigate the properties of dense suspensions and sediments of small spherical silt particles by means of a combined molecular dynamics and stochastic rotation dynamics (SRD) simulation. We include van der Waals and effective electrostatic interactions between the colloidal particles, as well as Brownian motion and hydrodynamic interactions which are calculated in the SRD part. We present the simulation technique and first results. We have measured velocity distributions, diffusion coefficients, sedimentation velocity, spatial correlation functions, and we have explored the phase diagram depending on the parameters of the potentials and on the volume fraction.
\end{abstract}

DOI: 10.1103/PhysRevE.72.011408

PACS number(s): 82.70.-y, 47.11.+j, 05.40.-a, 02.70.Ns

\section{INTRODUCTION}

We simulate claylike colloids, for which in many cases the attractive van der Waals forces are relevant. They are often called "peloids" (Greek: claylike). The colloidal particles have diameters in the range of some $\mathrm{nm}$ up to some $\mu \mathrm{m}$. In general, colloid science is a large field, where many books have been published [1-6]. The term peloid originally comes from soil mechanics, but particles of this size are also important in many engineering processes. Our model system of $\mathrm{Al}_{2} \mathrm{O}_{3}$-particles of diameter $0.5 \mu \mathrm{m}$ suspended in water is an often used ceramics and plays an important role in technical processes. In soil mechanics [7] and ceramics science [8], questions on the shear viscosity and compressibility as well as on porosity of the microscopic structure which is formed by the particles, arise $[9,10]$. In both areas, usually high volume fractions $(\Phi>20 \%)$ are of interest. The mechanical properties of these suspensions are difficult to understand. Apart from the attractive forces, electrostatic repulsion strongly determines the properties of the suspension. Depending on the surface potential, which can be adjusted by the $p \mathrm{H}$ value of the solvent, one can either observe the formation of clusters or the particles are stabilized in suspension and do sediment only very slowly. Hydrodynamic effects are also important for sedimentation experiments. Since typical Peclet numbers are of order 1 in our system, Brownian motion cannot be neglected.

In summary, there are many important factors which have to be included in a model which describes peloids in a satisfying way. Such a model is needed to gain a deeper understanding of the dynamics of dense colloidal suspensions. A lot of effort has been invested by applying different simulation methods, which have their inherent strengths but also some disadvantages. Simplified Brownian dynamics (BD), such as in the work of Hütter [11], does not contain longranged hydrodynamic interactions among particles at all. The computational cost is low, since hydrodynamics is reduced to a simple Stokes force and thus large particle numbers can be handled. BD with full hydrodynamic interactions utilizes a mobility matrix which is based on the Oseen- or Rotne-
Prager-Yamakawa tensor approximations which are exact in the limit of zero Reynolds number and zero particle volume fraction $[12,13]$.

This technique faces the main problem that the computational effort scales with the cube of the particle number due to the inversion of matrices.

The lattice Boltzmann (LB) method, on the other hand, is numerically efficient and intrinsically contains hydrodynamic interactions. Ladd and Verberg give an overview over the LB method and describe how to include stress fluctuations [14]. Adhikari et al. add noise to their model by introducing a noise term for every lattice velocity and node [15]. However, the discussion about the correct inclusion of thermal fluctuations is still ongoing $[15,16]$. Pair-Drag simulations have been proposed by Silbert et al. [17], which include hydrodynamic interactions in an approximative way. They have focused on suspensions with high densities (up to $50 \%$ ) of uncharged spherical colloidal particles. Here we use stochastic rotation dynamics (SRD) $[18,19]$, a recently developed method to simulate fluid flow, and combine this with a molecular dynamics (MD) simulation for the suspended particles. SRD is a particle-based method which does not show any numerical instabilities, contains thermal fluctuations intrinsically, and is simple to implement. Many important issues in fluctuation fluid dynamics such as sedimentation [20], vesicles in flow [21], polymers in flow [22], and reacting fluids [23] have been addressed very recently using this method. In this paper, first we discuss the main points of the MD part of our simulation code. Second we present the SRD method in the context of our work, and then we describe two alternative ways of coupling the two parts of the simulation and point out the advantages and disadvantages of these two possibilities. After that, we analyze the time scales which are relevant for the peloids, we want to simulate. Based on the insights of this section we show in the following section how to determine the simulation parameters. Then we describe how we have tested our simulation code and present first results in the following section. Finally in the last section we draw a conclusion and summarize shortly the model we have presented. 


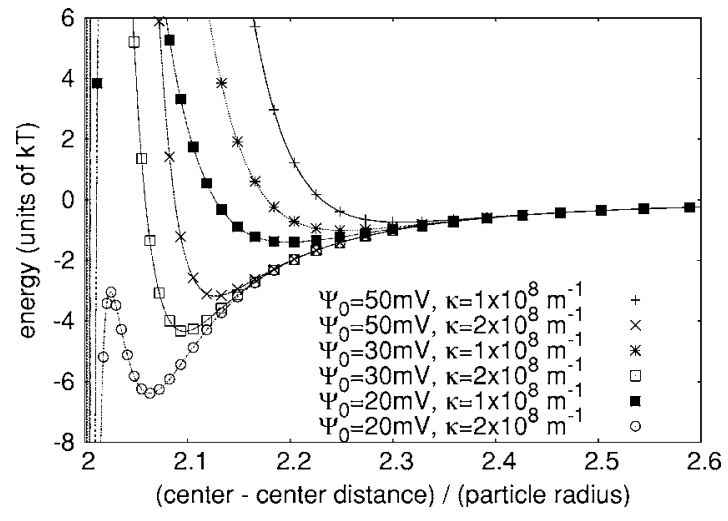

FIG. 1. DLVO potentials for $\mathrm{Al}_{2} \mathrm{O}_{3}$ spheres of $R=0.5 \mu \mathrm{m}$ diameter suspended in water. These are typical potentials used for our simulations as described below. The primary minimum at $d / R$ $=2.0$ is not reproduced correctly by the DLVO theory. It has to be modeled separately. In most of our cases the existence of the secondary minimum determines the properties of the simulated system.

\section{MOLECULAR DYNAMICS}

The colloidal particles in our simulation are represented by three-dimensional spheres. In order to correctly model the statics and dynamics when approaching stationary states, realistic potentials are needed. The interaction between the particles is described by Derjaguin-Landau-Verwey-Overbeek (DLVO) theory $[10,11,24]$. If the colloidal particles are suspended in a solvent, typically water, ions move into solution, whereas their counter-ions remain in the particle due to a different resolvability. Thus, the colloidal particle carries a charge. The ions in solution are attracted by the charge on the particles and form the electric double layer. It has been shown (see [24]) that the resulting electrostatic interaction between two of these particles can be described by an exponentially screened Coulomb potential

$$
V_{\text {Coul }}=\pi \epsilon_{r} \epsilon_{0}\left[\frac{4 k_{\mathrm{B}} T}{z e} \tanh \left(\frac{z e \Psi_{0}}{4 k_{\mathrm{B}} T}\right)\right]^{2} \frac{d^{2}}{r} \exp (-\kappa[r-d]),
$$

where $d$ denotes the particle diameter and $r$ is the distance of the particle centers. $z$ is the charge of the ions, $e$ is the elementary charge, $T$ is the temperature, $\Psi_{0}$ denotes the effective surface potential, and $\kappa$ is the inverse Debye screening length. In addition the behavior is determined by the attractive van der Waals interaction which can analytically be integrated over the two spheres. This leads to the second part of the DLVO potential:

$$
V_{\mathrm{VdW}}=-\frac{A_{\mathrm{H}}}{12}\left[\frac{d^{2}}{r^{2}-d^{2}}+\frac{d^{2}}{r^{2}}+2 \ln \left(\frac{r^{2}-d^{2}}{r^{2}}\right)\right] .
$$

$A_{\mathrm{H}}$ is the Hamaker constant which involves the polarizability of the particles and of the solvent. The DLVO potentials are plotted in Fig. 1 for six typical examples with different depths of the secondary minimum. The primary minimum has to be modeled separately, as discussed below.
To avoid the particles penetrating each other, one needs a repulsive force depending on their overlap. We are using a Hertz force described by the potential

$$
V_{\text {Hertz }}=K(d-r)^{5 / 2} \quad \text { if } r<d,
$$

where $K$ could be expressed by the elastic modulus of $\mathrm{Al}_{2} \mathrm{O}_{3}$. This would determine the simulation time step, but to keep computational effort relatively small, we determine the time step using the DLVO potentials as described later on and then choose a value for $K$. Two aspects have to be considered: $K$ has to be big enough, so that the particles do not penetrate each other by more than approximately $10 \%$ and it may not be too big, so that numerical errors are kept small, which is the case when the collision time is resolved with about 20 time steps. Otherwise total energy and momentum are not conserved very well in the collision.

Since DLVO theory contains the assumption of linear polarizability, it holds only for large distances-i.e., the singularity when the two spheres touch-does not exist in reality. Nevertheless, there is an energy minimum about $30 k_{\mathrm{B}} T$ deep, so that particles which come that close would very rarely become free again. To obtain numerical stability of our simulation, we model this minimum by a parabolic potential, some $k_{\mathrm{B}} T$ deep (e.g., $6 k_{\mathrm{B}} T$ ). The depth of the minimum in our model is much less than in reality, but the probability for particles to be trapped in the minimum has to be kept low enough so that only few of them might escape during simulation time.

The long-range hydrodynamic interaction is taken into account in a separate simulation for the fluid as described below. This can only reproduce interactions correctly down to a certain level. On shorter distances, a lubrication force has to be introduced explicitly in the molecular dynamics simulation as described in [25]. The most dominant mode, the socalled squeezing mode, is an additional force

$$
\mathbf{F}_{\text {lub }}=-\left(\mathbf{v}_{\text {rel }}, \hat{\mathbf{r}}\right) \hat{\mathbf{r}} \frac{6 \pi \eta r_{\mathrm{red}}^{2}}{r-r_{1}-r_{2}},
$$

with

$$
r_{\text {red }}=\frac{r_{1} r_{2}}{r_{1}+r_{2}},
$$

between two spheres with radii $r_{1}$ and $r_{2}$ and the relative velocity $\mathbf{v}_{\text {rel }} \cdot \eta$ is the dynamic viscosity of the fluid. $\mathbf{F}_{\text {lub }}$ diverges if particles touch each other. Therefore, we limit the force by introducing a minimum radius, where the force reaches its largest allowed value. The potential is shifted accordingly to smaller particle distances, so that the maximum force is reached for particles touching each other.

The Hertz force also contains a damping term in the normal direction,

$$
\mathbf{F}_{\text {Damp }}=-\left(\mathbf{v}_{\text {rel }}, \hat{\mathbf{r}}\right) \hat{\mathbf{r}} \beta \sqrt{r-r_{1}-r_{2}},
$$

with a damping constant $\beta$, and for the transverse direction a viscous friction proportional to the relative velocity of the particle surfaces is applied. 
For the integration of the translational motion we utilize a velocity Verlet algorithm (see [26], Chap. 3.2.1) to update the velocity and position of particle $i$ according to the equations

$$
\begin{gathered}
\mathbf{x}_{i}(t+\delta t)=\mathbf{x}_{i}(t)+\delta t \mathbf{v}_{i}(t)+\delta t^{2} \frac{F_{i}(t)}{m}, \\
\mathbf{v}_{i}(t+\delta t)=\mathbf{v}_{i}(t)+\delta t \frac{F_{i}(t)+F_{i}(t+\delta t)}{2 m} .
\end{gathered}
$$

For the rotation, a simple Euler algorithm is applied:

$$
\begin{gathered}
\omega_{i}(t+\delta t)=\omega_{i}(t)+\delta t \mathbf{T}_{i}, \\
\theta_{i}(t+\delta t)=\theta_{i}(t)+F\left(\theta_{i}, \omega_{i}, \delta t\right),
\end{gathered}
$$

where $\omega_{i}(t)$ is the angular velocity of particle $i$ at time $t, \mathbf{T}_{i}$ is the torque exerted by noncentral forces on the particle $i, \theta_{i}(t)$ is the orientation of particle $i$ at time $t$, expressed by a quaternion, and $F\left(\theta_{i}, \omega_{i}, \delta t\right)$ gives the evolution of $\theta_{i}$ of particle $i$ rotating with the angular velocity $\omega_{i}(t)$ at time $t$.

The concept of quaternions [26] is often used to calculate rotational motions in simulations, because the Euler angles and rotation matrices can easily be derived from quaternions. Using Euler angles to describe the orientation would give rise to singularities for the two orientations with $\theta= \pm 90^{\circ}$. The numerical problems related to this fact and the relatively high computational effort of a matrix inversion can be avoided using quaternions.

We have switched off dissipative forces and checked if the total energy and each component of the total momentum are conserved. We have verified this for the molecular dynamics simulation for the simulation of the fluid and for the coupled simulation separately.

We also checked that our implementation of the molecular dynamics code is correct by simulating eight large particles with Hertz repulsion and Coulomb friction in a closed box at a volume fraction of $\Phi \approx 20 \%$. We checked that the collisions are realistic-i.e., that the individual angular velocities for two particles interacting in a noncentral collision before and after they have touched are consistent.

\section{STOCHASTIC ROTATION DYNAMICS: SIMULATION OF THE FLUID}

The stochastic rotation dynamics method introduced by Malevanets and Kapral $[18,19]$ is a promising tool for a coarse-grained description of a fluctuating solvent, in particular for colloidal and polymer suspensions. The method is also known as "real-coded lattice gas" [27] or as "multi particle-collision dynamics" (MPCD) [28]. It can be seen as a "hydrodynamic heat bath," whose details are not fully resolved but which provides the correct hydrodynamic interaction among embedded particles [29]. SRD is especially well suited for flow problems with Peclet numbers of order 1 and Reynolds numbers on the particle scale between 0.05 and 20 for ensembles of many particles. ${ }^{1}$ The numerical effort scales linearly with the number of embedded colloidal particles unlike in Brownian dynamics, and only one random number per node (for the choice of the rotation matrix) is needed in contrast to fluctuating lattice Boltzmann. For Peclet numbers of order 1, about 3-5 SRD particles are required per box (or node) whose positions and velocities can be seen as the degrees of freedom in that node. In three dimensions (3D) this amounts to $18-25$ variables per node which is similar to the 15 or 19 speed lattice-Boltzmann method.

While the LB method might be slower than SRD in the regime of large thermal fluctuations it has the advantage that it can be used for almost arbitrarily high Peclet numbers just by reducing the amplitude of the noise. To reduce the noise in SRD, a huge number of fluid particles per node has to be used which makes the method inapplicable at Peclet numbers higher than about 20. Fortunately it has been shown by Padding and Louis [20] that basic properties of sedimentation such as the main settling speed are hardly affected by thermal noise.

The method is based on so-called fluid particles with continuous positions and velocities which follow a simple, artificial dynamics.

The system is coarse grained into cubic cells of a regular lattice with no restriction on the number of particles in a cell. The evolution of the system consists of two steps: streaming and collision. In the streaming step, the coordinate of each particle is incremented by its displacement during the time step. Collisions are modeled by a simultaneous stochastic rotation of the relative velocities of every particle in each cell. The dynamics is explicitly constructed to conserve mass, momentum, and energy, and the collision process is the simplest consistent with these conservation laws. It has been shown that there is an $H$ theorem for the dynamics and that this procedure yields the correct hydrodynamic equations for an ideal gas [18].

Consider a set of $N$ point particles with (continuous) coordinates $\mathbf{r}_{i}(t)$ and velocities $\mathbf{v}_{i}(t)$. In the streaming step, all particles are propagated simultaneously by a distance $\mathbf{v}_{i} \tau$, where $\tau$ is the value of the discretized time step. For the collision step, particles are sorted into cells, and they interact only with members of their own cell. Typically, the simplest cell construction consisting of a hypercubic grid with mesh size $a$ is used. The collision step consists of an independent random rotation of the relative velocities $\mathbf{v}_{i}-\mathbf{u}$, of the particles in each cell, where the macroscopic velocity $\mathbf{u}(\xi, t)$ is the mean velocity of the particles in the cell with coordinate $\xi$. The local temperature $T(\xi, t)$ is defined via the meansquare deviation of the particle velocities from the mean ve-

\footnotetext{
${ }^{1}$ For low Peclet numbers Brownian motion dominates and sedimentation takes place very slowly. The simulations require a huge number of time steps. Then Brownian simulation (BS), including short-range hydrodynamics interactions, might be a more suitable tool, since not the complete velocity field has to be calculated. For very high Peclet numbers, SRD becomes inefficient due to the necessary averaging. For high Reynolds numbers a small time step and high spatial resolution would be necessary, which increases the computational effort extremely.
} 
locity in a cell. All particles in a cell are subject to the same rotation, but the rotation angles of different cells are statistically independent. There is a great deal of freedom in how the rotation step is implemented [18,30], since, by construction, the local momentum and kinetic energy are invariant. The dynamics is therefore summarized by

$$
\begin{gathered}
\mathbf{r}_{i}(t+\tau)=\mathbf{r}_{i}(t)+\tau \mathbf{v}_{i}(t) \\
\mathbf{v}_{i}(t+\tau)=\mathbf{u}\left[\xi_{i}(t+\tau)\right]+\omega\left[\xi_{i}(t+\tau)\right]\left\{\mathbf{v}_{i}(t)-\mathbf{u}\left[\xi_{i}(t+\tau)\right]\right\}
\end{gathered}
$$

where $\omega\left(\xi_{i}\right)$ denotes a stochastic rotation matrix and $\xi_{i}$ is the coordinate of the cell occupied by particle $i$ at the time of the collision. $\mathbf{u}(\xi) \equiv(1 / M) \Sigma_{k \in \xi} \mathbf{v}_{k}$ is the mean velocity of the particles in cell $\xi$. $\omega$ is taken to be a rotation by an angle $\pm \alpha$, with probability $1 / 2$. We are using rotations about the three coordinate axes with $\alpha= \pm 90^{\circ}$, because these are the most simple rotation matrices one can imagine in $3 \mathrm{D}$, since they only contain entries taken out of $\{0, \pm 1\}$. This has been suggested by Tuzel et al. [31]. In every time step for each cell one of these 6 possibilities is chosen with equal probability $1 / 6$. However, any stochastic rotation matrix consistent with detailed balance can be used.

In order to remove low temperature anomalies and to achieve exact Galilean invariance, we use a modification of the original algorithm [30]: all particles are shifted by the same random vector with components in the interval $[-a / 2, a / 2]$ before the collision step. Particles are then shifted back by the same amount after the collision. The random vectors of consecutive iterations are uncorrelated. Ihle and Kroll have discussed in Refs. [32,33] why this simple procedure works and showed that it leads to transport coefficients independent of an imposed homogeneous flow field. In Refs. [34,35] analytical calculations of the transport coefficient in this method are presented.

Two different methods to couple SRD and MD simulations have been introduced in the literature. We have implemented them both, and we are using them depending on what we plan to measure. The first one [27] is much more accurate in resolving the local velocity field around the colloidal particles. Lubrication effects are reproduced well by this coupling method. The second one [36] resolves the velocity field only down to a length scale of the particle diameter. On the other hand, the method becomes much faster because of the lower resolution. In both coupling methods the long-range hydrodynamic interactions are reproduced.

\section{COUPLING I: PLACING FLUID PARTICLES OUTSIDE OF COLLOIDAL PARTICLES}

In the combined MD and SRD simulation the fluid particles have to interact with the colloidal particles and transfer momentum from one to the other part of the simulation. One possibility to do this is, as suggested by Inoue et al. [27], to check after each streaming step of a fluid particle $i$ if its new position $x_{i}(t+\tau)$ is within a colloidal particle and, if yes, to modify its position and velocity. In this coupling step total momentum has to be conserved, which means, that when modeling the "collision" between the fluid particle and the colloidal particles, one has to make sure that the change of momentum of the fluid particle is transfered to the suspended particle. The calculations described in the following are done in a frame fixed on the colloid particle.

One can think of several different methods to assign a new position to the fluid particle, which have been shown to work properly.

(i) Place it on the shortest distance to the surface of the colloidal particle and move it with its new velocity half of a time step.

(2) Calculate the point and the exact time when the fluid particle has entered into the colloidal particle and move it back to there. Then choose a new velocity and move the fluid particle with the new velocity for the remainder of the time step.

Both methods turned out to work, where the second one is more accurate but more computationally intensive as well. Just to place the fluid particle directly on the surface and move it again in the next time step turned out to produce an increase of the fluid particle density around the colloidal particle. Anomalies in the fluid temperature could also be found when the fluid particles were placed directly on the colloid surface.

To increase stability of the simulation the idea is not to conserve energy in every single collision, but to use a thermostat and choose the new velocities according to a given distribution. The new velocities should point from the colloid surface to the outer area. Since the interior of the colloidal particle usually does not contain any fluid particles and the velocity distribution next to a colloidal particle should be independent of neighboring particles, the velocity distribution for the newly chosen fluid particle velocities has to be the same as if the space inside the suspended particle was filled with fluid particles. Assume these imaginary fluid particles having the same density and temperature as in the remainder of the fluid bath. Then, one could evaluate the velocity distribution for the reflected fluid particles by taking the velocity distribution of the imaginary fluid particles passing through the colloid surface. But it is a nontrivial task to analytically calculate this distribution for a spherical area. However, if the mean free path of the fluid particles is small compared to the diameter of the colloidal particles, we can safely assume the colloid surface to be an infinitely extended plane separating the space into two regions [27]. Then one finds the following distribution:

$$
\begin{gathered}
p\left(v_{n}\right) \sim v_{n} \exp \left(-\beta v_{n}^{2}\right), \\
p\left(v_{t}\right) \sim \exp \left(-\beta v_{t}^{2}\right),
\end{gathered}
$$

with

$$
\beta=\frac{m_{f}}{2 k_{\mathrm{B}} T},
$$

where $v_{n}$ is the normal component and $v_{t}$ is the tangential component of the fluid particle velocity in the frame fixed to the surface of the large particle. $m_{f}$ is the mass of a fluid particle. In the following sections we describe how $m_{f}$ has to 
be chosen. $T$ is the temperature to which this thermostat is adjusted, and the whole system will adopt this temperature after a transient time. The tangential component can be obtained by computing $\sqrt{x_{1}^{2}+x_{2}^{2}}$ of two independent and Gaussian distributed random variables.

Since the fluid particles of the SRD are artificial particles within the context of this mesoscopic simulation method, their mean free path and their momentum are different from the corresponding values for single solvent molecules. Because of this, there is a depletion force acting on colloidal particles which is much larger than in reality. Depletion forces are only relevant in systems with very big molecules-e.g., polymer solutions with added small particles or binary mixtures of particles with clearly separated diameters. There, each of the small particles carries a considerable momentum - which is also the case in the SRD simulations. Nevertheless, unrealistically high depletion forces can be suppressed by reflecting fluid particles many times: If after the collision step the fluid particle is placed in another colloidal particle, the collision step is repeated for that colloidal particle and so on, until the fluid particle reaches a position outside any colloidal particle or until a maximum number $N_{\max }$ of collisions has been calculated through. We have measured the depletion force and found out that a limit of $N_{\max } \sim 10$ is a good compromise between computational speed and accuracy. The depletion force does not decay substantially stronger if the limit is increased, but the computational effort still grows with $N_{\max }$ (at most linearly), because some fluid particles are trapped in a small gap between two colloidal particles and jump from one to the other. This in fact would still decrease the depletion force, but in the meantime the calculation for the remaining system is interrupted until finally eventually one single fluid particle is reflected the very last time. It is obvious that this scenario can easily be truncated. The remaining depletion force can be neglected at least in the cases where strong attractive van der Waals forces or strong repulsive electrostatic forces are present.

\section{COUPLING II: ROTATING VELOCITIES OF THE COLLOIDAL PARTICLES}

A second possibility to couple SRD and MD simulations is to sort the colloidal particles into the SRD boxes and include their velocity in the rotation step. This technique has been used to model protein chains suspended in a liquid $[36,37]$. The mean velocity in each cell has then to be weighted with the mass of the particle (because the mass of colloid particles differs at least by one order of magnitude from the one of the fluid particles and their inertia dominates the flow field next to it). The calculation of $\mathbf{u}\left[\xi_{i}(t+\tau)\right]$ in Eq. (12) is modified to

$$
\mathbf{u}(\xi) \equiv \frac{1}{M} \sum_{k \in \xi} \mathbf{v}_{k} m_{k},
$$

where we sum over all colloid and fluid particles in the cell. $m_{k}$ is the mass of the particle with index $k$ and $M \equiv \Sigma_{k \in \xi} m_{k}$ is the total mass contained in the cell.
The coupling acts on the center of mass of the colloidal particles and affects only the fluid particles within the same cell. This means, to affect the same area of the flow field like in reality, one has to choose the cells to be of the same size as the colloidal particles. Obviously, the mesh size is drastically larger than in the first coupling method and the flow field cannot be resolved in detail. The fact that colloidal particles push away the solvent as well as depletion and lubrication forces cannot be reproduced at any level.

\section{TIME-SCALE ANALYSIS}

Our system contains many different, let us say $L$, time scales, which differ by several orders of magnitude making brute force numerical simulations very time consuming or even impossible. These time scales can be used to define $L$ -1 dimensionless characteristic numbers, such as the Reynolds or Peclet number, as the ratio of two time scales. If one can manage to adjust the simulation parameters such that all these characteristic numbers are the same as in the experiment, the simulations should be able to exactly reproduce the dynamical behavior of the real system. Of course, therefore one has to change quantities like the temperature or the viscosity of the fluid.

Often, it is sufficient to reproduce only a few of all characteristic numbers exactly-i.e., only those which are believed to be significant for the behavior. For example, in sedimentation processes where the Reynolds number is much smaller than unity, it may be modified to another value, which still fulfills the condition of being much smaller than 1. In both cases the Stokes limit is a valid approximation. As a general rule of thumb, dimensionless numbers of order 1 are important to be reproduced since they represent two competing dynamical effects. The reason to modify the other "insignificant" numbers is to reduce the ratio of the largest to the smallest time scale which determines the numerical effort. In order to decide which are the dimensionless numbers that can be safely modified without changing the physics too much, a detailed analysis of the different time scales is needed.

We start with the largest scales. After some time an isolated spherical particle sedimenting in a liquid reaches the so-called Stokes velocity

$$
v_{S}=\frac{2}{9} \frac{R^{2} g}{\nu}\left(\frac{\rho_{m}}{\rho_{w}}-1\right)
$$

$\nu$ is the kinematic viscosity, $g$ denotes gravity, $\rho_{m}$ is the mass density of the particle, and $\rho_{w}$ the mass density of the solvent. This velocity is obtained from the force balance between buoyancy and weight of the particle, $F_{G}=4 \pi\left(\rho_{m}\right.$ $\left.-\rho_{w}\right) g R^{3} / 3$, and the drag force in a viscous liquid, $F_{D}$ $=6 \pi \nu \rho_{w} R v$.

The drag force $F_{D}$ also defines the mobility $\mu=v / F_{D}$ $=1 /\left(6 \pi \nu \rho_{w} R\right)$ of a spherical particle. The time for a particle to move a distance of its diameter, $2 R$, is denoted by 


$$
\tau_{S}=\frac{2 R}{v_{S}}=\frac{9 \nu}{\operatorname{Rg}\left(\frac{\rho_{m}}{\rho_{w}}-1\right)} .
$$

By means of the Einstein relation $D=\mu k_{\mathrm{B}} T$ we obtain the diffusion constant $D$ for the particle,

$$
D=\frac{k_{\mathrm{B}} T}{6 \pi \nu \rho_{w} R} .
$$

The mean-square displacement of a diffusing particle in each dimension $i$ is given by

$$
\left\langle x_{i}^{2}(t)\right\rangle=2 D t
$$

hence, the time the particle needs to diffuse a distance of $2 R$ is of the order of

$$
\tau_{D}=\frac{2 R^{2}}{D}=\frac{12 \pi \nu \rho_{w} R^{3}}{k_{\mathrm{B}} T},
$$

which we call the diffusion time.

The ratio $\tau_{D} / \tau_{S}$ measures the importance of Brownian motion in the system and is called the Peclet number, Pe $=\tau_{D} / \tau_{S}$. It turns out to be close to unity here. Inserting the definitions for $\tau_{D}$ and $\tau_{S}$, one notices that Pe depends on the fourth power of the radius $R$,

$$
\mathrm{Pe}=\frac{v_{S} R}{D}=\frac{F_{G} R}{k_{\mathrm{B}} T}=\frac{4 \pi g R^{4}\left(\rho_{m}-\rho_{w}\right)}{3 k_{\mathrm{B}} T} .
$$

Let us consider another time proportional to $\tau_{D}$ : we assume a regular three-dimensional, cubic array of spheres which are separated by gaps of size $R / 2$. Then, the volume concentration of this suspension is in the intermediate regime, $\phi$ $=0.268$. The time one sphere diffuses the distance of a gap is given by $\tau_{G}=\tau_{D} / 16$.

Now, let us discuss another important time called the particle relaxation time, which is related to how long it takes the particle to react to an imposed force; i.e., this time measures the inertia effects. Consider Newton's equation for a particle of mass $m$ subject to a force $F$ and a friction coefficient $\xi$, $m \partial v / \partial t=-\xi v+F$. Expanding the velocity $v$ around the stationary state, $v=v_{S}+\delta v$, gives

$$
\frac{\partial \delta v}{\partial t}=-\frac{\xi}{m} \delta v
$$

which leads to an exponential decay on a time scale $\tau_{P}$ $=m / \xi$. Identifying the friction $\xi$ with $1 / \mu$ and inserting the mass leads to

$$
\tau_{P}=\frac{2}{9} \frac{R^{2}}{\nu} \frac{\rho_{m}}{\rho_{w}} .
$$

Now we consider a very short time scale $\tau_{F}$; the time fluid momentum diffuses a distance $2 R$-i.e., $(2 R)^{2}=2 \nu \tau_{F}$ leading to

$$
\tau_{F}=\frac{2 R^{2}}{\nu}
$$

which helps defining the particle Reynolds number as

$$
\operatorname{Re}=\frac{\tau_{F}}{\tau_{S}}=\frac{R v_{S}}{\nu} .
$$

Finally, we have to discuss another important short length scale due to a short-range potential among the colloidal particles. This scale usually determines the maximum time step in molecular dynamics. Guided by the analogy to a harmonic oscillator with frequency $\omega=\sqrt{k / m}$, we replace the spring constant $k$ with the second derivative of the interparticle potential $\partial^{2} V(R) / \partial R^{2}$ and use the period of this oscillation to define the interaction time scale,

$$
\tau_{V}=\frac{2 \pi}{\omega}=2 \pi \sqrt{\frac{m l^{2}}{A_{H}}},
$$

where we approximate the derivative of the potential by means of the Hamaker constant $A_{H}$ as a typical size of the potential and a typical distance $l$ such as the distance between the surface of the particle and the primary potential minimum due to the combined effect of van der Waals attraction and screened Coulomb repulsion. Comparison of $\tau_{V}$ and $\tau_{P}$ can answer the question whether the oscillations of two particles around the primary or secondary minimum are visible or whether the creeping or overdamped case is realized where friction is dominating over inertia. Analyzing a harmonic oscillator with damping constant $\xi$ one finds that creeping being established at

$$
\tau_{P} \leqslant \frac{\tau_{V}}{4 \pi}
$$

In using this relation a lubrication force described in Eq. (4) has to be taken into account. This force is proportional to the difference of normal velocities of two approaching particles, and in this sense it can be seen as an additional contribution to the friction coefficient. It becomes huge at short interparticle distances $d$, and it will turn out later that even without this addition all particles considered here are well inside the creeping regime due to the large friction in water. This is the justification that so far many people used Brownian dynamics for this system instead of molecular dynamics $[11,38]$. In our situation, including thermal fluctuations and full hydrodynamics consistently is easier to do in molecular dynamics. Moreover, with our parameters the MD is at least competitive or even faster than previous BD calculations.

\section{SIMILARITY CONSIDERATIONS AND DETERMINATION OF SIMULATION PARAMETERS}

\section{A. Introduction}

The determination of parameters for a mesoscopic model to quantitatively compare with experiment is a nontrivial task. Typical values of the parameters in an experiment are listed in Table I. For these values of the parameters in the experiment all the time scales defined in the previous section are calculated and listed in Table II. This tells us that the Peclet number is $\mathrm{Pe}=\tau_{D} / \tau_{S}=0.74$ and we have a competition between convection due to gravity and Brownian motion. The particle Reynolds number is very small-i.e., $\mathrm{Re}$ $=\tau_{F} / \tau_{S}=4.0 \times 10^{-7}$. The ratio of $\tau_{V}$ to $\tau_{P}$ is larger than $4 \pi$; 
TABLE I. Parameters for the simulation.

\begin{tabular}{cc}
\hline \hline Particle radius $R$ & $0.4 \mu \mathrm{m}$ \\
Temperature $T$ & $300 \mathrm{~K}$ \\
Mass density of particle $\rho_{m}$ & $3.9 \times 10^{3} \mathrm{~kg} / \mathrm{m}^{3}$ \\
Mass density of water $\rho_{w}$ & $1.0 \times 10^{3} \mathrm{~kg} / \mathrm{m}^{3}$ \\
Boltzmann constant $k_{\mathrm{B}}$ & $1.38 \times 10^{-23} \mathrm{~J} / \mathrm{K}$ \\
Kinetic viscosity of water $\nu$ & $10^{-6} \mathrm{~m}^{2} / \mathrm{s}$ \\
Gravity $g$ & $9.81 \mathrm{~m} / \mathrm{s}^{2}$ \\
Hamaker constant $A_{H}$ of $\mathrm{Al}_{2} \mathrm{O}_{3}$ in $\mathrm{H}_{2} \mathrm{O}$ & $4.76 \times 10^{-20} \mathrm{~J}$ \\
Distance to primary minimum $l$ & $0.008 \mu \mathrm{m}$ \\
\hline \hline
\end{tabular}

hence, oscillations of particles in their short-range potentials are overdamped, already without considering lubrication forces. We get $\tau_{P} \ll \tau_{G}$, since the particles are well relaxed before they hit each other due to Brownian motion. $\tau_{F} \ll \tau_{D}$; hence, the transport of momentum through the fluid is much faster than if transported directly by the particle. These are the dynamical characteristics which have to be preserved by any parameter changes; in particular, the Peclet number has to be kept exactly the same. Of course, the static properties such as the ratio of kinetic energy $\sim k_{\mathrm{B}} T$ to the potential energies, $\sim m g R$, and $\sim A_{H}$ have to be kept the same too.

However, using identical parameters as shown in Table I in an MD simulation would require of the order of $10 \tau_{S} / \tau_{P}$ $\approx 5 \times 10^{7}$ iterations to see sufficient progress in the sedimentation process. This is an unacceptably high numerical effort, which must be reduced without significantly changing the physics of this process. First, we now show how to choose the parameters for a simulation using the coupling method I. After that, we describe what has to be changed using the coupling II.

\section{B. Determination of the parameters for coupling I}

We start by choosing reasonable parameters for the hydrodynamic part of the code-i.e., stochastic rotation dynamics-since this is time consuming and the most storage intensive part of our simulation. For the moment we keep the particle radius constant at $R=0.4 \mu \mathrm{m}$. Let $a$ be the lattice constant of the SRD grid. By choosing $a=R / 2$ a spherical particle covers about 34 boxes which is a sufficient resolution of the particle. We get $a=0.2 \mu \mathrm{m}$.

We use an average number of $M=2.5$ fluid particles per box, which leads to $6 M=15$ real numbers (3 velocity and 3 position coordinates in 3 dimensions) to be stored for every box. A larger $M$ would reduce Brownian motion and increase CPU-time and storage requirements. Using a smaller number leads to a very long effective mean free path of the fluid

TABLE II. Time scales which arise in a system characterized by the parameters listed in Table I.

\begin{tabular}{cccccc}
\hline \hline$\tau_{S}$ & $\tau_{D}$ & $\tau_{G}$ & $\tau_{V}$ & $\tau_{F}$ & $\tau_{P}$ \\
\hline $0.791 \mathrm{~s}$ & $0.582 \mathrm{~s}$ & $36.5 \mathrm{~ms}$ & $7.45 \mu \mathrm{s}$ & $0.320 \mu \mathrm{s}$ & $0.139 \mu \mathrm{s}$ \\
\hline \hline
\end{tabular}

particles (sometimes there is only one particle per box and no collision takes place), which results in a large viscosity and a bad resolution of the flow field around the colloidal particles.

Next, we choose the ratio of the mean free path $\lambda$ $=\tau \sqrt{k_{\mathrm{B}} T / m_{f}}$ to the lattice constant. $m_{f}$ is the mass of the fluid particle and $T$ the effective temperature of the fluid particles which can differ by several orders of magnitude from the real temperature of the experiment as will be explained later. In Ref. [30] it was discovered that a ratio $\lambda / a$ smaller than 0.5 leads to anomalies in the model, which can be corrected by a random shift of the lattice prior to every rotation. Here, we set $\lambda=0.6 a=0.12 \mu \mathrm{m}$ to have sufficient resolution of the flow and random shifts are not needed.

The rotation angle $\alpha$ is taken to be $90^{\circ}$ because this gives the most simple rotation matrix.

The exact expression for the shear viscosity for $\alpha=90^{\circ}$ is given by [34]

$$
\nu=\frac{a^{2}}{18 \delta t}\left(1-\frac{1-\mathrm{e}^{-M}}{M}\right)+\frac{k_{\mathrm{B}} T \delta t}{4 m_{f}} \frac{M+2}{M-1} .
$$

Inserting $M=2.5$ and expressing temperature by means of $\lambda$ it follows for our choice of parameters that

$$
\nu=0.3052 \frac{a^{2}}{\delta t} \text {. }
$$

In order to reproduce the same diffusion coefficient as seen in experiments, $\delta t$ has to be determined by means of the Einstein relation,

$$
D=k_{\mathrm{B}} T \mu=\frac{k_{\mathrm{B}} T}{6 \pi \nu \rho_{w} R} .
$$

Setting $\rho_{w}=M m_{f} / a^{3}$, using $\nu$ from Eq. (29), and expressing $k_{\mathrm{B}} T / m_{f}$ by means of $\lambda$ one finds $\delta t=0.025 a^{3} /(D R)$. Inserting the diffusion coefficient expected in reality from the Einstein relation, $D=5.49 \times 10^{-13} \mathrm{~m}^{2} / \mathrm{s}$, we arrive at a time step $\delta t$ $=0.91 \mathrm{~ms}$ for the SRD algorithm. This time step is of course too large to resolve the motion of colloidal particles due to interparticle forces and friction. Hence, a two-step method is needed: The trajectory for the colloidal particles is integrated by another, smaller time step $\delta t_{M}$. This also means that the extensive SRD procedure is only applied every $\left(\delta t / \delta t_{M}\right)$ th iteration of the MD algorithm, thus reducing the required computer power substantially.

The way parameters are derived implicitly means that we keep $\tau_{S}$ and $\tau_{D}$ as in reality. This corresponds to $\tau_{S} / 0.91 \mathrm{~ms}=869 \mathrm{SRD}$ iterations until a colloidal particle has fallen down by one diameter $2 R$ which is affordable. The kinematic viscosity in the simulation is much smaller than in nature $\left(\nu_{\text {model }}=1.34 \times 10^{-11} \mathrm{~m}^{2} / \mathrm{s}\right)$.

Next, one has to check what happens to the particle relaxation time $\tau_{P}$. The requirement is that it should be much larger than the one given in Table I (in order to increase numerical efficiency) and, on the other hand, it should still be smaller than $\tau_{G}$ to ensure that particles can relax between consecutive collisions caused by thermal motion. Following Eq. (23), we obtain $\tau_{P}=10.36 \mathrm{~ms}$. This is an acceptable value: it is much larger than the $0.139 \mu$ s seen in reality and 
still smaller than $\tau_{G}=36.5 \mathrm{~ms}$. Therefore it needs 3.5 SRD steps to relax a particle which means that the process still can be resolved.

Considering momentum transport in the fluid versus direct transport: During time $\tau_{D}$, momentum in the fluid is transported a distance $x^{2}=2 \nu \tau_{G}=24.43 a^{2}$, i.e., $x=4.94 a$ $=2.47 R$. Hence, momentum transport in the fluid is only slightly faster than by diffusive transport, which is still acceptable, even though in the real system it is much faster. This is reflected in a Reynolds number which is larger by a factor of $10^{-6} / \nu_{\text {model }}=0.746 \times 10^{5}$ in the simulation, i.e., $\operatorname{Re}$ $=3.0 \times 10^{-2}$. This again reflects the fact that the SRD model is efficient only if the Peclet and Reynolds numbers are in the range between 0.05 and 20 .

Now, the gravity constant $g$ of the model has to be determined requiring that the Stokes velocity be the same as given in Table I. Since thermal convection of the fluid is not important for our simulation, we can neglect gravity on the fluid particles. Therefore, there is no buoyancy force in the simulation. We can correct for that by assuming a smaller gravity constant modified by the density ratio of colloid material and fluid. We find

$$
g_{\text {model }}=g_{\text {real }} \frac{\nu_{\text {model }}}{\nu_{\text {real }}}\left(1-\frac{\rho_{w}}{\rho_{m}}\right)=9.78 \times 10^{-5} \mathrm{~m} / \mathrm{s}^{2} .
$$

As mentioned above, not only the viscosity, but also the temperature in our simulation may be different from the one in nature. To see that we calculate the ratio $\Lambda=\rho_{m} / k_{\mathrm{B}} T$. In nature we have $\Lambda=0.942 \times 10^{24} \mathrm{~s}^{2} / \mathrm{m}^{5}$. In the model we get $\Lambda_{\text {model }}=3.9 \mathrm{Mm}_{f} /\left(a^{3} k_{\mathrm{B}} T\right)$ where we express $k_{\mathrm{B}} T / m_{f}$ by means of the mean free path and the time step $\lambda^{2} /(\delta t)^{2}$. One finds that $\Lambda_{\text {model }}$ is scaled by a factor of $7.44 \times 10^{4}$. The static features have to be reproduced by the model, and therefore we have to keep the ratio of kinetic and potential energy $k_{\mathrm{B}} T / A_{H}$ constant. This means that the ratio $\rho_{m} / V_{\text {Pot }}$ and especially $\rho_{m} / A_{H}$ has also to be scaled by this factor. We use $A_{H}=4.76 \times 10^{-20} \mathrm{~J} /\left(7.44 \times 10^{4}\right)$ in the model, corresponding to new $A_{H}=6.39 \times 10^{-25} \mathrm{~J}$. From Eq. (26) we get a scaled $\tau_{V}$ of $2.03 \mathrm{~ms}$ corresponding to $\tau_{V} / \tau_{P}=0.196$ [which is smaller than $4 \pi$; see Eq. (27)]. The unscaled value is 53.6. The creeping case is restored by the lubrication force, which we have included in the MD simulation and which grows for smaller gaps between the particles. The lubrication force determines the small iteration time step $\delta t_{M}$ for the MD simulation. We chose $\delta t_{M}=2 \mu \mathrm{s}$, which is about 200 times larger than it would be if all the original parameters would have been kept and $\min \left(\tau_{V}, \tau_{P}\right)$, being much smaller, would determine the time step.

Comparing to the SRD time step we see that every 455 small steps one SRD step is performed. We need 869 SRD steps and $4 \times 10^{5} \mathrm{MD}$ steps to see a colloidal particle sinking

TABLE III. Time scales in the simulation using coupling method I.

\begin{tabular}{cccccc}
\hline \hline$\tau_{S}$ & $\tau_{D}$ & $\tau_{G}$ & $\tau_{V}$ & $\tau_{F}$ & $\tau_{P}$ \\
\hline $0.791 \mathrm{~s}$ & $0.582 \mathrm{~s}$ & $36.5 \mathrm{~ms}$ & $2.03 \mathrm{~ms}$ & $22.9 \mathrm{~ms}$ & $10.36 \mathrm{~ms}$ \\
\hline \hline
\end{tabular}

TABLE IV. Time scales in the simulation using coupling method II.

\begin{tabular}{cccccc}
\hline \hline$\tau_{S}$ & $\tau_{D}$ & $\tau_{G}$ & $\tau_{V}$ & $\tau_{F}$ & $\tau_{P}$ \\
\hline $0.791 \mathrm{~s}$ & $0.582 \mathrm{~s}$ & $36.5 \mathrm{~ms}$ & $1.56 \mathrm{~ms}$ & $14.0 \mathrm{~ms}$ & $6.02 \mathrm{~ms}$ \\
\hline \hline
\end{tabular}

down by one diameter. The time scales in the simulation are summarized again in Table III.

\section{Determination of the parameters for coupling II}

To simulate the same system with coupling method II, we use the same particle radius $R=0.4 \mu \mathrm{m}$. The lattice constant has now to be chosen differently because the colloidal particles are coupled to the SRD simulation as mass points. They have influence on the fluid which is in the same cell, and therefore the size of the cell can be understood as the volume within which the SRD simulation "feels" the colloidal particles and we choose the lattice constant in a way that the volume of the cell is equal to the volume of a colloidal particle: $a=6.25 \times 10^{-7} \mathrm{~m}$. A smaller lattice constant in this context would model smaller colloidal particles in the SRD part of the simulation. The velocity field would be resolved better, but since coupling method II does not allow a resolution smaller than the colloidal particles, one cannot expect to gain any information from the fluid simulation on smaller length scales than the colloidal particle size. Any attempt to increase the resolution of the SRD simulation would only cause a larger computational effort.

Since we do not modify the Peclet number, we have to choose approximately the same number of fluid particles per colloidal particle. Since the box size has increased with respect to the coupling method I, we have to assume more particles per box now. We choose $M=60$ (which would correspond to two particles per box in the coupling method I, but since the boxes are much larger now, we can slightly reduce the ratio of fluid particles per colloidal particle).

We choose $\lambda / a=0.5$ and use random grid shifts here to avoid the fluid particles interacting too often with the same partners which causes artifacts in their correlation. The rotation angle $\alpha$ is again $90^{\circ}$ to achieve very simple matrices. Following the same procedure as for coupling method I [Eq. (28)] we find a time step for the SRD of

$$
\delta t=2.05 \mathrm{~ms} .
$$

According to Eq. (29) the viscosity in the simulation results to $\nu_{\text {model }}=2.29 \times 10^{-11} \mathrm{~m}^{2} / \mathrm{s}$. The gravity constant therefore has to be rescaled by a factor of 58813 , and the temperature and potentials have to be scaled by $43733 .^{2}$

The resulting characteristic times are shown in Table IV. $\tau_{S}$ and $\tau_{D}$ are again kept as in reality. Now we need $\tau_{S} / 2.05 \mathrm{~ms}=385 \mathrm{SRD}$ iterations until a colloidal particle has fallen down by one diameter, which is much faster than by using coupling method I. $\tau_{P}$ is still smaller than $\tau_{G}$. Again $\tau_{P}$

\footnotetext{
${ }^{2}$ Where we take care of the fact that we do not apply gravity to the fluid particles.
} 
became larger than $\tau_{V}$, so that we have to include a lubrication force in the MD part for coupling II as well. Apart from that, the order of the different time scales is reproduced again: $\tau_{P}<\tau_{F}<\tau_{G}<\tau_{D}<\tau_{S}$.

Momentum is transported 1.34 times faster in the fluid as by the particles themselves. Short-range hydrodynamic interactions which cannot be resolved are in this sense comparable to particle-particle collisions whereas for long-range interactions the slightly faster transport of momentum can reproduce coarse-grained hydrodynamic effects. Again, to model these effects comparable to reality, the Reynolds number has to be much smaller than unity. We find $\mathrm{Re}=1.77$ $\times 10^{-2}$.

If we include lubrication forces in the MD simulation in order to reproduce at least to some extend short-range hydrodynamics, we have to choose the same MD time step as for coupling method I but we need approximately 50\% less CPU time for the hydrodynamics. Even though it seems to be a too simplified approach, we can reproduce a volumefraction-dependent sedimentation velocity as will be described in Sec. X.

\section{SIMULATION SETUP}

\section{A. Boundary conditions}

Most simulations have been performed using periodic boundary conditions in all three directions. Then the total momentum may not change in any simulation step if no external forces (like gravity) are applied. If gravity on the colloidal particles is applied in a system with periodic boundary conditions, this would accelerate the whole system, since the total force on the center of mass is not vanishing. In a real system there is friction at the walls and, even more important, there is an equilibrium between hydrostatic pressure acting on the surface of a given volume and gravity acting as a body force. Since we simulate a volume in the center of the suspension, we either have to apply the pressure on the walls or, which is easier, make sure that in sum the forces on the center of mass of the whole simulated system vanishes. Therefore, we follow the center of mass; i.e., on particles with higher density their gravity minus buoyancy has to be applied, so that they move downward whereas the same force in opposite direction has to be applied to the fluid, which makes it move upward like in a sedimentation vessel with a closed bottom.

For the following discussion we define that the direction in which eventually gravity is applied is called the $-z$ direction if a shear force is applied acts in the $x$ direction. Using closed boundaries wall effects may be introduced; e.g., crystallization starts earlier than in the bulk. This effect could be observed especially when gravity was switched off and only closed-boundary conditions were applied. This is a finite-size effect, which is not that strong if periodic boundaries are applied. But in the case of gravity being applied, the whole system accelerates. To face this problem, three possibilities were tested.

(i) Fix the boundaries only in the $z$ direction.

(ii) Fix the boundaries in the $x$ and $y$ directions and apply a no-slip condition for the fluid. (iii) Choose periodic boundaries in all directions and compensate the gravitation on the colloidal particles with a force in the opposite direction applied on the fluid.

Possibilities (i) and (ii) simulate a system close to a wall; in case (i), it is the bottom of a vessel, whereas in case (ii) the experiment would be done in a capillary. Possibility (iii) turned out to be the most realistic simulation, although it can start to drift if the compensating force is not adjusted accurately. Slowly accumulated drifts of the center of mass can be removed every hundreds of SRD time steps if necessary.

\section{B. Temperature and thermostat}

We have measured the temperature of the colloidal particles for different setups. If damping constants are chosen appropriately, the resulting temperature fits very well the temperature, which we have adjusted for the fluid by the initial conditions. If we additionally switch on a thermostat which we describe in the following, the measured temperature exactly agrees with temperature adjusted by the thermostat. When gravity is applied to the system, particles are accelerated and if in addition periodic boundaries are used, a thermostat is absolutely needed to remove the extra energy, introduced by the periodic boundary in $z$ direction in combination with gravity.

Therefore we use a modified version of the thermostat described in [26] (Chap. 7.4.1, p. 227f). The thermostat, originally suggested by Heyes [39], chooses a random scaling factor $\zeta$ for the velocities from an interval $[1-\gamma, 1+\gamma]$. The scaling of the velocity is then accepted or rejected according to a Monte Carlo scheme. However, the detailed balance is not fulfilled for the choice of $\zeta$ described in [26]. In our implementation of the thermostat, we randomly choose an $\epsilon$ in the interval $[0, \gamma]$ and apply for $\zeta$ one of the values $1+\epsilon$ or $1 /(1+\epsilon)$, each of them with the probability of $\frac{1}{2}$. With one of these values the velocity is scaled by the Monte Carlo acceptance rate. Also the temperature in our case is defined slightly different from [26]: the mean velocity u within one SRD cell defines the velocity field of the fluid and gives the hydrodynamic interaction between the colloidal particles. Therefore it may not be modified by the thermostat. We only scale the velocity component relative to the mean velocity: $\mathbf{v}_{i}^{\text {new }}=\zeta\left(\mathbf{v}_{i}-\mathbf{u}\right)+\mathbf{u}$. The Monte Carlo acceptance rate in our case is given by

$$
\zeta^{(3(M-1))} \exp \left(-(M-1)\left(\zeta^{2}-1\right) T / T^{*}\right),
$$

with

$$
T=\frac{m_{f}}{2(M-1) k_{\mathrm{B}}} \sum_{i=1}^{M}\left(\mathbf{v}_{i}-\mathbf{u}\right)^{2},
$$

which is the local temperature in the SRD cell and $T^{*}$ denotes the temperature to which the thermostat will drive the system. $M$ is the number of particles in the cell. Note that one has to divide the total thermal energy in the SRD cell by $M-1$ instead of $M$ to calculate the local temperature. This reflects the fact that the mean velocity $\mathbf{u}$ in the cell already contains three degrees of freedom which the particles in the SRD cell have. The choice of $\gamma$ and the frequency with 
which the thermostat is called to work determine the relaxation rate, with which the system adapts $T^{*}$. The version described in [26] shows deviations of the achieved temperature for small numbers of particles per cell, whereas our implementation exactly reproduces $T^{*}$. The thermostat can even be extended to particles of different mass-i.e., colloid and fluid particles where the mass is used as weight factor for all velocities of the simulation.

\section{Outlook: Shear}

There are several possibilities to shear the system. If one only has MD particles, one can use moving walls either with a spring constant and a friction coefficient or with direct hard reflections, where a moving wall is assumed and the reflection is calculated in the moving frame fixed to the wall.

These approaches of course neglect all effects (like pseudo wall slip), which appear close to a wall in a shear experiment with a suspension. There, shear stress has to be applied to the fluid which then drags the suspended particles. One way to implement this is to add a small velocity offset to all fluid particles which are reflected. Since this approach works well and the colloidal particles are dragged by the fluid, we apply shear in this way to our system.

\section{TESTS OF THE SIMULATION CODE}

\section{A. Conservation of energy, velocity distributions}

We have checked that the total energy is conserved in the molecular dynamics simulation if all damping constants are switched off. Otherwise or if the total energy even increases in spite of damping constants, the MD time step has been chosen too large. In the SRD simulation energy is conserved as well and if we use coupling method II also for the total system energy is conserved within numerical accuracy. With coupling method I (where a thermostat is already included in the coupling method) or if we switch on an additional thermostat, energy will not exactly be conserved but the system will reach a stable-i.e., equilibrated-state. In that sense, total energy (including thermal energy) will converge to a constant value.

In SRD simulations without any embedded particles, the total energy contains only the kinetic energy of the fluid particles. It is fully determined by the initialization of the particle velocities. We can choose three uniformly distributed random numbers to initialize the three velocity components for the fluid particles. In thermal equilibrium the distribution should be a Gaussian, which in fact can be observed in our simulations after some tens of SRD time steps. If colloidal particles are included into the system, they should reach a thermal equilibrium, at least as long as no external forces are applied. Damping terms would reduce fluctuations, so to check if the colloidal particles reach the same temperature as the fluid particles, damping constants have to be set to zero. Both distributions are shown in Fig. 2. They are both Gaussian with the correct temperature, even though for initialization uniformly distributed random numbers (square well) had been used. The tests are performed with both coupling methods. We have carried out simulations with particle radii of
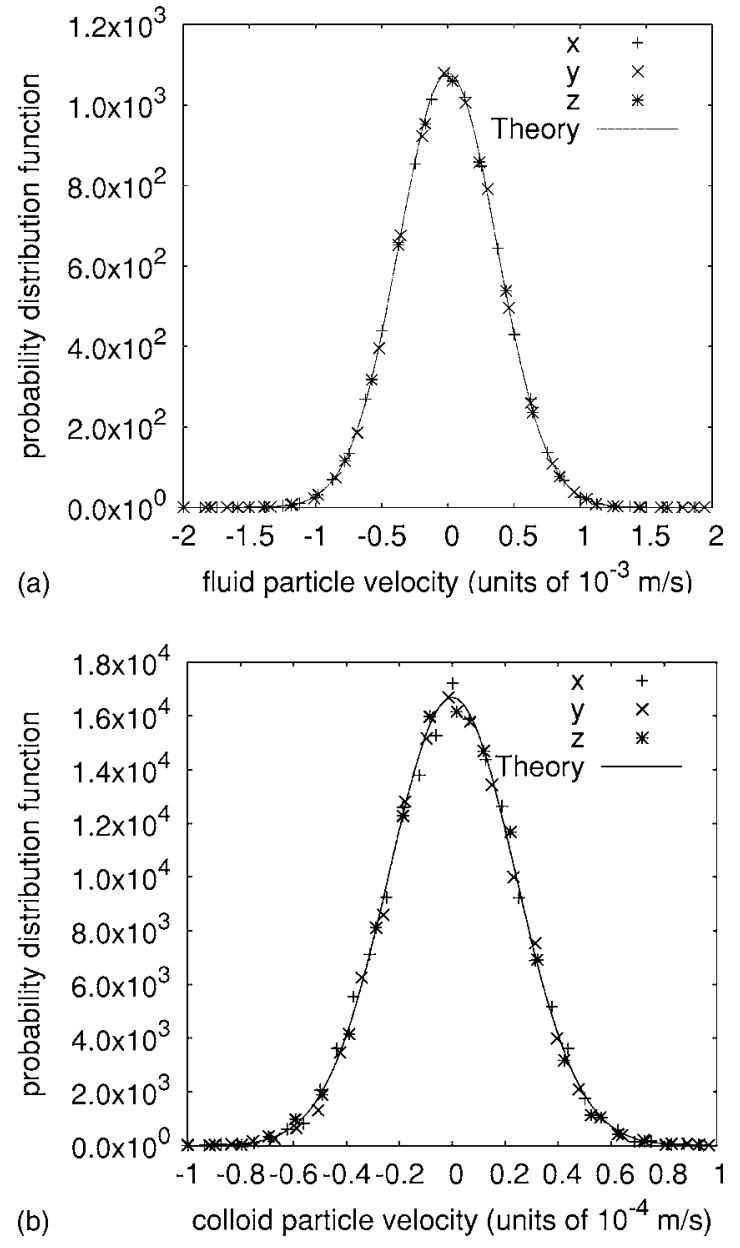

FIG. 2. Velocity distribution of fluid (a) and colloid (b) particles in a SRD simulation after thermalization. Particle density is $3900 \mathrm{~kg} / \mathrm{m}^{3}$, the time step $2.0 \mu \mathrm{s}$, model temperature $T$ $=10.57 \mathrm{mK}$, fluid particle mass $m_{f}=1.0667 \times 10^{-18}$, and particle diameter $d=0.5 \mu \mathrm{m}$. The theoretical Gaussian curve is plotted as well as the measured velocity distributions.

$0.4 \mu \mathrm{m}$ and $0.25 \mu \mathrm{m}$, where the Peclet number (for the simulations where gravity is applied) is 0.11 and, of course, it takes much longer to observe sedimentation.

\section{B. Viscosity}

The diffusion coefficient of suspended colloidal particles can be used to check if the desired viscosity could really be achieved in the simulation. Using Eq. (18) we can, once we have measured $D$, calculate the kinematic viscosity $\nu$ and compare it to the value we have used to determine the simulation parameters like the SRD time step. We achieve a deviation of less than $20 \%$ in a diluted system compared to the theoretical value for an infinitely diluted system. Note that $D$ is a fixed number only in the limit of an infinitely diluted system and only if the interaction potentials between the colloidal particles are exclusively repulsive.

We are using two different methods, either the GreenKubo method or direct evaluation of the mean-square displacement. The first is even very accurate, if only few particles are used, but consumes much computer time and 
memory because all particle velocities have to be stored for all time steps used in the calculation. That means, for higher volume fractions, it is more efficient just to sum up all the mean-square displacements within a given period of time. To calculate $D$ using the Green-Kubo method one uses the following relation:

$$
\begin{gathered}
g_{x}(j)=\lim _{I \rightarrow \infty} \frac{1}{I M_{\mathrm{Tot}}} \sum_{i=1}^{I} \sum_{n=1}^{M_{\mathrm{Tot}}} v_{x, n}((i+j) \delta t) v_{x, n}(i \delta t), \\
D_{x}=\delta t\left(\frac{1}{2} g_{x}(0)+\sum_{j=1}^{\infty} g_{x}(j)\right),
\end{gathered}
$$

where $M_{\text {Tot }}$ is the total number of particles in the system and $I$ is the number of time steps used to calculate the contribution $g(j) . v_{x, n}(i \delta t)$ denotes the $x$ component of the velocity of particle $n$ in the $i$ th time step. The sum in the expression for $D_{x}$ is in principle an infinite one, but since the contributions $g(j)$ decay with $j^{-3 / 2}$, one can truncate this sum after some tens of terms. $D_{y}$ and $D_{z}$ can be calculated accordingly. In Fig. 3(a) we show the diffusion coefficient in each direction. In numerical calculations it is impossible to evaluate an infinite sum. In Eq. (34), I is limited at least by the total number of time steps within the simulation and in Eq. (35) the sum therefore is not infinite either. Since the contributions $g_{x}(j)$ become more and more inaccurate for larger $j$ we truncate the sum after $n$ terms and find that in our simulations for $n \approx 50$ the diffusion coefficient does not change anymore in a systematic way if $n$ is increased further. In Fig. 3(b) the last term of the sum is shown. For larger values, they fluctuate due to the finite sum in Eq. (34) which leads to the inaccuracy in the right part of Fig. 3(a). These fluctuations become smaller for longer simulation runs, but do not change the value of the diffusion coefficient taken as an average from the center part of Fig. 3(a). ${ }^{3}$

For the mean-square displacement in one direction during a time interval $\Delta t$ we calculate

$$
D_{x}=\frac{1}{2 \Delta t M_{\text {Tot }}} \sum_{i=1}^{M_{\text {Tot }}}\left[x_{i}(t+\Delta t)-x_{i}(t)\right]^{2}
$$

and $D_{y}$ and $D_{z}$ accordingly. For medium densities we have compared both methods and achieved the same results within error bars. Depending on the number of particles, we use one of both methods.

According to Richardson and Zaki [40], the mean sedimentation velocity of particles suspended in a liquid depends on the volume fraction $\phi$ as

$$
v_{s}(\phi)=v_{\infty}(1-\phi)^{l},
$$

with a typical exponent $l$ between $\approx 2.5$ and 4 depending on the boundary conditions. For periodic boundary conditions, Peclet number of $\mathrm{Pe}=1$, and Reynolds number $\mathrm{Re} \ll 1$ we find an exponent of 3.5 (Fig. 4) even when we use coupling method II, where only the long-range hydrodynamic interaction can be calculated correctly. A similar value is found for

${ }^{3}$ This can be seen as a smooth cutoff of the sum in Eq. (35).
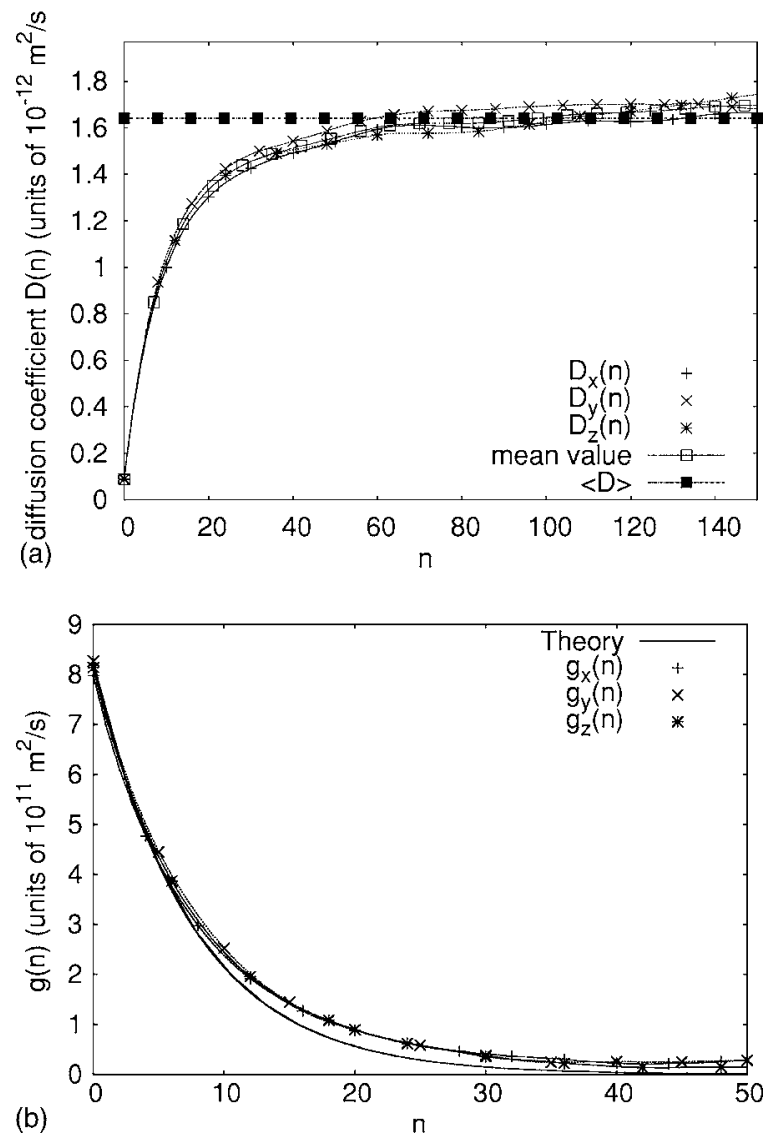

FIG. 3. (a) Evaluation of $D$ using the Green-Kubo method: the plot shows the sum of $\sum_{j=1, \ldots, n} g_{x, y, z}(j)$ and the estimated $D$. (b) The decay of the contributions $g_{x, y, z}(j)$. We have measured the diffusion constant of soft spheres coupled with coupling II to the SRD at low volume fractions.

$\mathrm{Pe}=2$ and $\mathrm{Pe}=\frac{1}{2}$. Padding and Louis have found that the exponent $l$ depends very weakly on the Peclet number [20]. We have used here coupling method II, but some investigations have also been carried out using coupling method I. In the first view there is no big difference apparent between the two coupling methods, at least as long as, like in this test of our simulation code, no attractive forces are included. Our first results where we have studied the peloid system in more detail are presented in the following section.

\section{RESULTS}

\section{A. Spatial correlation functions}

For our production runs, we have simulated volume fractions of $7 \%, 14 \%, 21 \%, 28 \%$, and $35 \%$ in a cube with an extension of $(6 \mu \mathrm{m})^{3}$. Therefore are $231,462,693,924$, and 1155 colloidal particles, respectively, and $2.0 \times 10^{5}$ fluid particles necessary.

We have evaluated the particle-particle correlation function. For attractive potentials several sharp peaks can be observed and we assign them to distinct local orders of particles. Oscillations can be found in the correlation function. They are caused by exclusion of volume. In the case of at- 


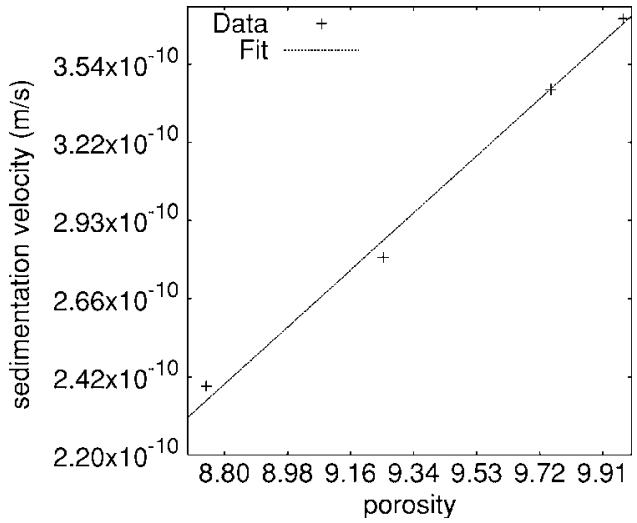

FIG. 4. Mean sedimentation velocity over porosity $(1-\phi)$ according to Eq. (37): measured values and fit curve in a log-log-plot. The Peclet number of this simulation is 1 . Coupling method II has been used for this plot.

tractive forces they are less pronounced than if mainly repulsive interaction is present; see Figs. 5 and 6.

In Fig. 5 the particles cluster due to their attractive potentials and form stable configurations. The diameter of the particles is $5 \times 10^{-7} \mathrm{~m}$. There is a sharp peak in the spatial correlation function of the particle centers at exactly that distance $2 R$, where two particles touch each other in the very left part of the plot (A). Then, for larger particle separation, the correlation function starts to grow and drops suddenly after a peak at $1 \mu \mathrm{m}(\mathrm{D})$, which is twice the diameter $(4 R)$. This is the contribution of two particles touching the same third particle. The distance between them depends on the angle which they form with the particle in the middle, but it is at last twice the diameter, when they are in a straight line, which explains the sudden drop of the correlation function. If several particles stick together, the straight line is stabilized. This explains the peak at the end of this section of the correlation function.

Two more peaks can clearly be assigned to configurations: One of them is from two particles touching two other par-

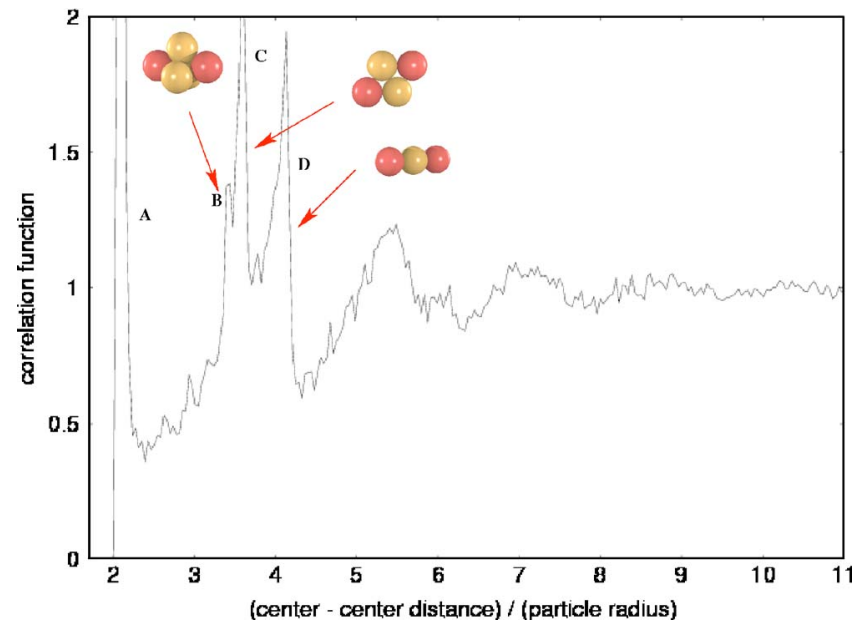

FIG. 5. (Color online) Correlation function of $\mathrm{Al}_{2} \mathrm{O}_{3}$ for $\Psi_{0}$ $=50 \mathrm{mV}$ and $\kappa=3 \times 10^{8} / \mathrm{m}$. The potential is attractive; thus, peaks (labeled by letters) can be identified and assigned to special local configurations (see text).

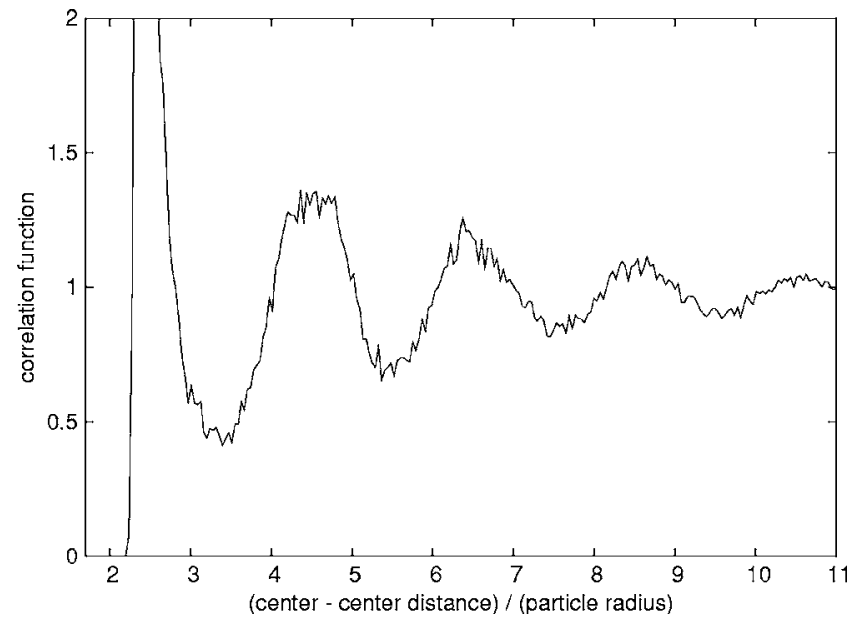

FIG. 6. Correlation function of $\mathrm{Al}_{2} \mathrm{O}_{3}$ for $\Psi_{0}=50 \mathrm{mV}$ and $\kappa$ $=7.3 \times 10^{7} / \mathrm{m}$. Repulsive potentials. One can see oscillations caused by the excluded volume.

ticles, which themselves touch each other (C). There again the case of all particles being in the same plane can be stabilized by other particles surrounding them. The particles under consideration are then separated by a distance of $2 R \sqrt{3}$. But of course, bending this configuration is still a degree of freedom which brings the two particles slightly closer to each other. Thus their contribution to the correlation function is shifted downward. The fourth peak at $\frac{4}{3} R \sqrt{6}$ reflects two particles, both touching three particles, which themselves are touching each other and define a plane (B). There is no freedom anymore for the two particles touching all the three of them at the same time. One can place one of them at one side of the plane and the other one at the other side.

When the potentials are mainly repulsive and the minimum caused by the van der Waals attraction is only a fraction of $k_{\mathrm{B}} T$, the spatial correlation function looks completely different, as depicted in Fig. 6: The peaks described in the previous paragraphs have disappeared here. The primary peak has moved to a slightly larger distance, since the repulsive potential hinders the particles from touching each other.

In Fig. 7 we compare the correlation function of Fig. 6 with the potential used for that simulation. The maximum of the correlation function coincides with the minimum of the potential, but as the minimum is not very sharp, the particles are not restricted to fixed geometries and are in a steady process of rearrangement which results in broader peaks. This process could also be studied by evaluating the velocity correlation function for the colloidal particles which is related to the viscosity of the sample. The correlation of particles which are several diameters apart is still remarkable, as it is transmitted by the particles in between. The oscillations of the correlation function can be understood as a formation of layers where the probability of finding a particle in a certain layer is higher than in between.

\section{B. Shear}

We have carried out simulations with shear and gravity. For the particles the boundaries in the $z$ direction were closed 


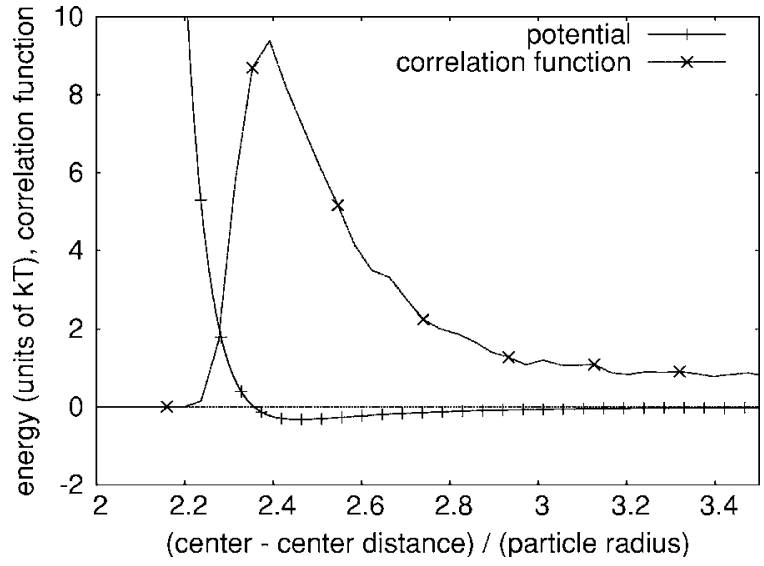

FIG. 7. Plot of the correlation function of Fig. 6 together with the potential used in this simulation. One can see that the maximum of the correlation function occurs for the distance, at which the very shallow secondary minimum of the potential is located.

and gravity was applied in the negative $z$ direction only to the colloidal particles. For the fluid particles the boundary in the $z$ direction was closed as well and additionally a velocity offset was added to apply a shear in the $x$ direction. Boundaries for fluids and for particles were periodic in the $x$ and $y$ directions. Velocity distribution functions have been evaluated. For the cases we investigated, after a transient they are all Gaussian (Fig. 8).

\section{Phase diagram}

We have explored the phase diagram for $\mathrm{Al}_{2} \mathrm{O}_{3}$ with respect to screening length and effective surface potential. We could identify the regions of suspended single particles and of flocculation (Fig. 9). The transition between these two regions depends on both parameters: the Debye screening length and effective surface potential. It is known that the $p \mathrm{H}$ value determines the effective surface potential $\Psi_{0}$ and that the salt concentration and $p \mathrm{H}$ value determine the Debye screening length $\kappa[9]$. Exact relations between salt concentration and $p \mathrm{H}$ value on the one side and $\kappa$ and $\Psi_{0}$ on the other side are not known a priori for the parameter ranges of

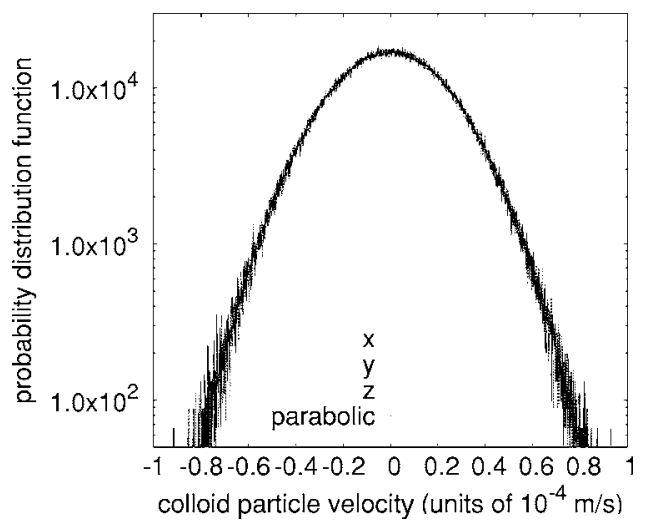

FIG. 8. Velocity distribution of colloidal particles for each direction. Semilogarithmic plot where deviations from a Gaussian would be visible by deviations from a parabolic profile.

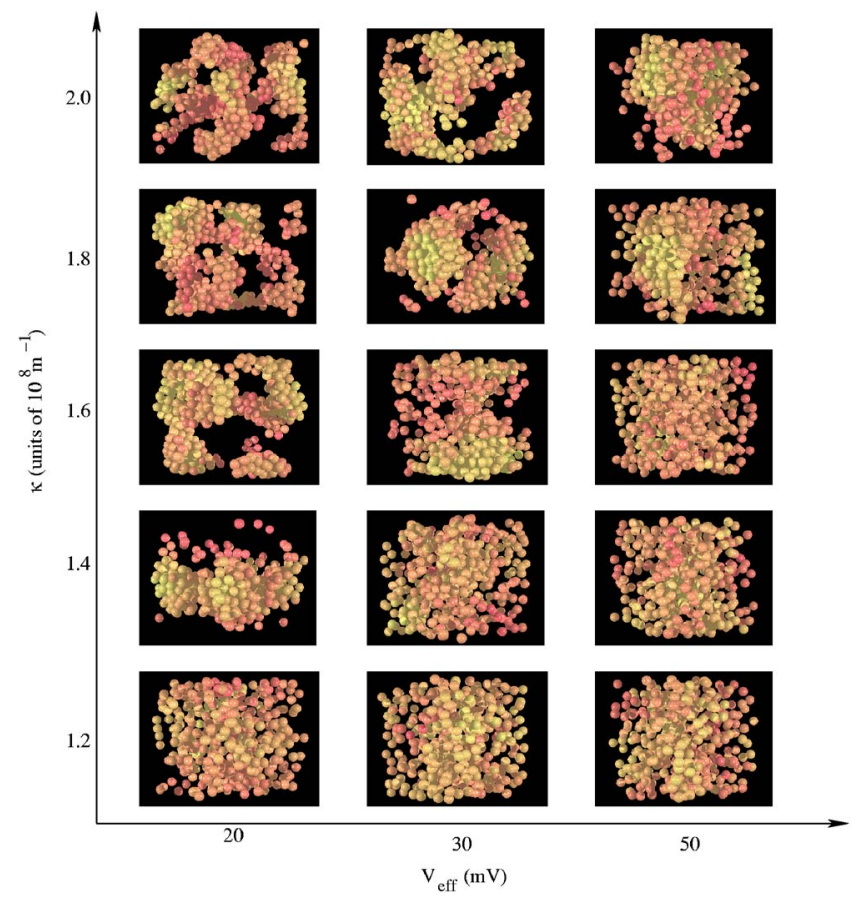

FIG. 9. (Color online) Snapshots from the phase diagram of $\mathrm{Al}_{2} \mathrm{O}_{3}$ : For the DLVO potentials with different effective surface charge and different screening length one can either observe cluster formation or single particles in suspension. The simulation was done at room temperature for $1 \mathrm{~s}$ of real time and a particle diameter of $0.5 \mu \mathrm{m}$. Gravity has not been applied here. The pictures are corresponding to the values written on the axis. For this figure we have chosen the simulation runs for $14 \%$ volume fraction with 462 colloidal particles.

our suspensions. There are approximations for very diluted systems and low salt concentrations. It is known that for $\mathrm{Al}_{2} \mathrm{O}_{3}$ the surface potential becomes zero for $p \mathrm{H} \approx 8.7$ [41]. However, a phase transition between clustering in the upper left part of Fig. 9 and a suspended regime in the lower right part can be found in the simulations in analogy to the experi-

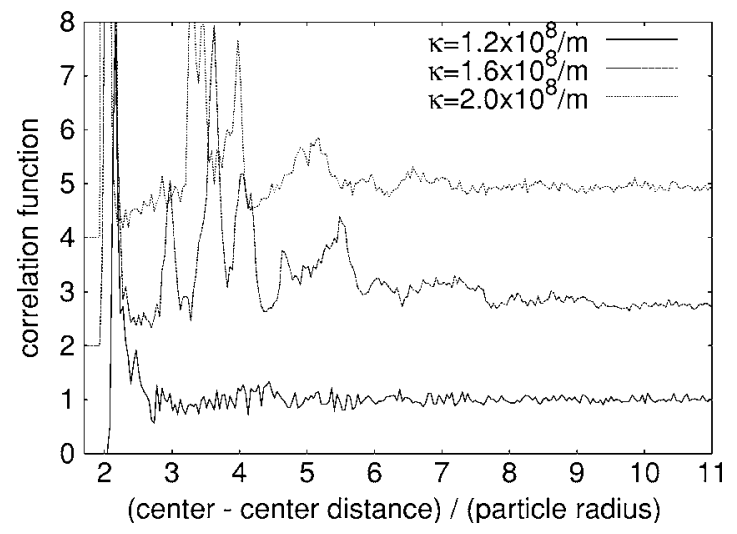

FIG. 10. Correlation function and its dependence on the inverse Debye screening length $\kappa . \Psi_{0}=20 \mathrm{mV}$ and $\Phi=0.14$ have been kept constant. For shorter Debye screening lengths the attractive force becomes stronger and leads to clustering, which is reflected in the appearance of peaks. The single curves have been shifted with respect to each other. 


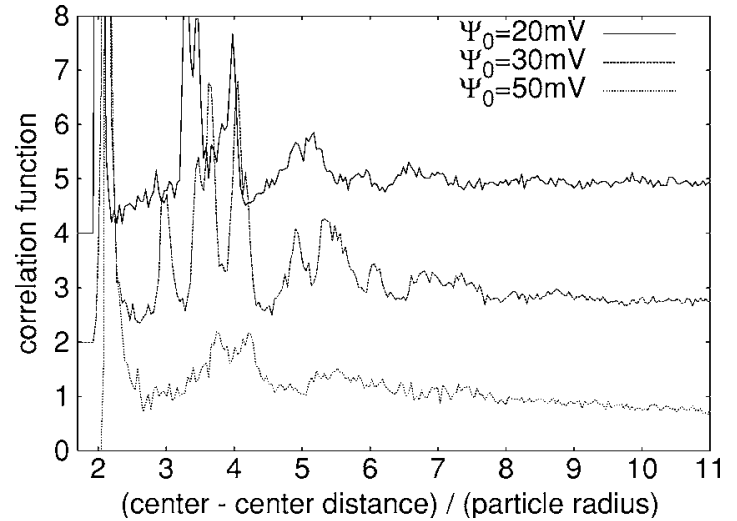

FIG. 11. Correlation function and its dependence on the effective surface potential $\Psi_{0} . \kappa=2 \times 10^{8} \mathrm{~m}^{-1}$ and $\Phi=0.14$ have been kept constant. The higher the effective surface potential, the stronger the attraction force, and clustering can be seen in the growing peaks.

ment. The spatial correlation function can be evaluated for all the simulated cases and it can be used as a tool to identify the two regions of the phase diagram.

Figures 10-13 show selected examples of correlation functions for different parameter sets. The first and second graphs refer to a volume fraction $\Phi=14 \%$ which also has been used for the phase diagram of Fig. 9. In Fig. 10 the correlation function has been plotted for every other image of the left column in the phase diagram in Fig. 9. One can see that for suspended particles only the first peak can be found in the correlation function. The secondary minimum in the potential causes the particles to glue for short times before they continue with their diffusion process. With increasing $\kappa$ the secondary minimum approaches the particle surface, and therefore the main peak is shifted to smaller distances. At the same time it becomes deeper so that clusters are formed and more peaks occur. The peak at a distance of $2 R \sqrt{2} \approx 3 R$ disappears again, when the attraction becomes stronger since this is a meta stable configuration of particles forming an octahedron. Figure 11 corresponds to the first

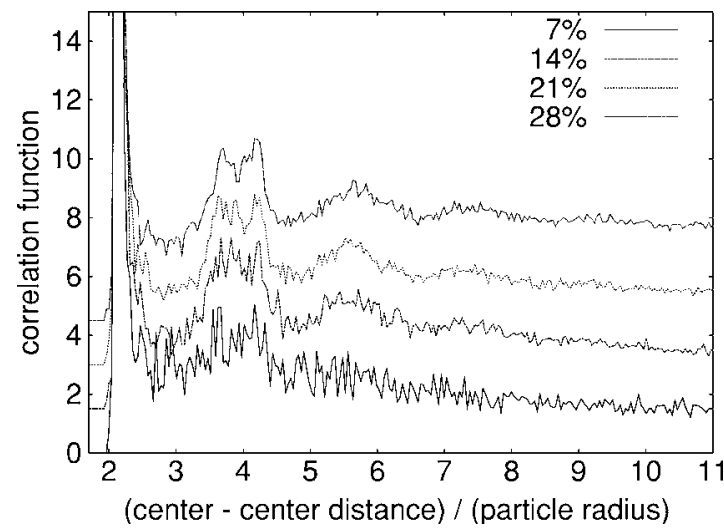

FIG. 12. Correlation function and its dependence on the volume fraction $\Phi$. Effective surface potential $\Psi_{0}=20 \mathrm{mV}$ and $\kappa=1.4$ $\times 10^{8} \mathrm{~m}^{-1}$ have been kept constant. For center-center distances between six and eight particle radii broad peaks start to appear for larger volume fractions.

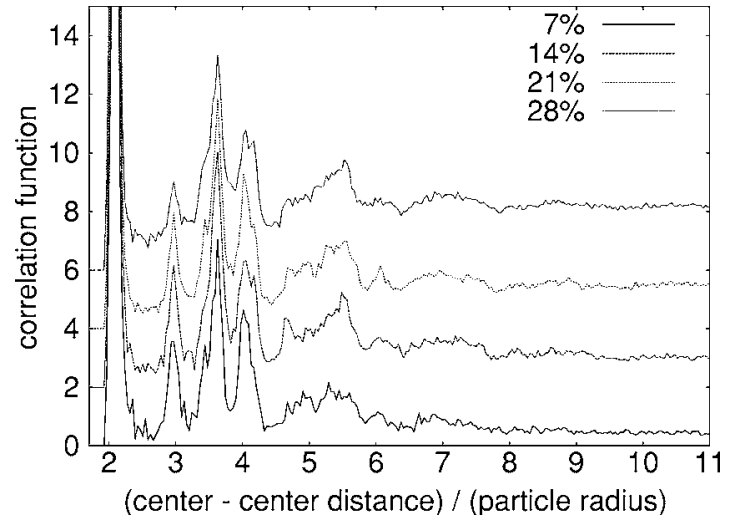

FIG. 13. Correlation function and its dependence on the volume fraction $\Phi$. Effective surface potential $\Psi_{0}=20 \mathrm{mV}$ and $\kappa=1.6$ $\times 10^{8} \mathrm{~m}^{-1}$ have been kept constant. Due to a small change in $\kappa$ with respect to Fig. 12, one can cross the phase border between suspended particles and clustering regime. Also here long-range correlations become more pronounced for high volume fractions.

row of images of Fig. 9. In this case the depth of the secondary minimum is adjusted by changing the effective surface potential. Again the transition between clustering regime and suspension can be observed. The potentials used here are among the ones plotted above in Fig. 1. ${ }^{4}$ In Figs. 12 and 13 the dependence of the correlation function on the volume fraction can be seen. In both cases long-range correlations become more pronounced with increasing volume fraction. This is shown for the suspended regime (Fig. 12) and for the clustering regime (Fig. 13), where the transition between the two cases presented here is achieved by a variation of $\kappa$ by only $10 \%$.

\section{Diffusion}

We measured the diffusion coefficient of colloidal particles with attractive potentials. In Fig. 14 we show the diffusion coefficient for $\mathrm{Al}_{2} \mathrm{O}_{3}$ with an effective surface potential of $\Psi_{0}=50 \mathrm{mV}$ and an inverse Debye screening length of $\kappa=2 \times 10^{8} \mathrm{~m}^{-1}$ for room temperature. One can see that the mobility of the particles decays since a cluster formation process takes place and the particles in the cluster are relatively fixed. The remaining mobility consists of two parts: Particles can still, with a nonvanishing probability, leave the cluster by thermal activation and the cluster itself can take part in a diffusion process; it can vibrate or be deformed-all of these are processes which are taking place on much longer time scales than the single-particle diffusion. By studying the dependence of the diffusion coefficient on the potentials and on the volume fraction, one might be able to find an answer to the question as to which of these processes is important for the dynamics of the system in which part of the phase diagram of Fig. 9.

\section{CONCLUSION}

We have shown that by combining a stochastic rotation dynamics and a molecular dynamics simulation it is possible

\footnotetext{
${ }^{4}$ In current simulations we did not yet distinguish between clustering in the primary or secondary minimum.
} 


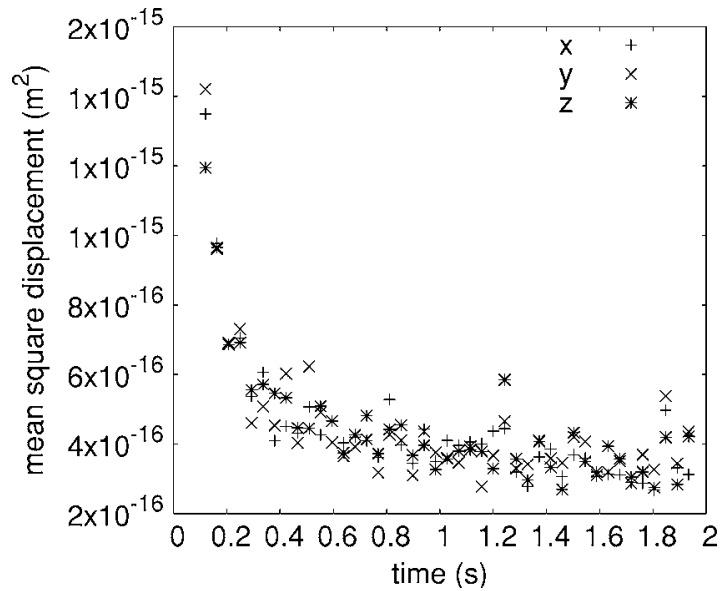

FIG. 14. Mean-square displacement (projection on each of the axis). For this simulation $\Psi_{0}=50 \mathrm{mV}$ and $\kappa=3 \times 10^{8} / \mathrm{m}$ have been used. First particles move by diffusion, are attracted, and then form clusters of larger size and lower mobility, which can be observed in a decay of the diffusion length for a given period of time. We have simulated 1155 particles in a cube with $6 \mu \mathrm{m}$ extension, which results in a volume fraction of $35 \%$.

to study dense colloidal suspensions. We have explained how to determine effective parameters for the simulation (box size $a$, simulation time step $\delta$, number of fluid particles per box $M$, etc.). It is possible to relate the simulation to very distinct experimental conditions since all parameters (density, temperature, potentials, etc.) which enter into the de- scription are scaled in a well-defined manner. We have presented first results which demonstrate the power of the model. We have demonstrated that the Richardson-Zaki law is reproduced already with the simple and fast coupling method II, and we have studied the dependence of the pair correlation function on the shape of the interaction potentials. We have shown how one can distinguish if for given Debye screening length $\kappa$, effective surface potential $\Psi_{0}$, and Hamaker constant $a_{\mathrm{H}}$ if the system is in the clustering or suspended regime.

We are planning to carry out detailed investigations of the properties described in the two preceding sections (diffusion coefficient, correlation functions, sedimentation velocity) as well as cluster size and shape. Then these quantities can be analyzed under shear, their dependence on the shear rate, and the shear viscosity of the suspension, containing the fluid and particles, which both contribute to a complex shear viscosity.

\section{ACKNOWLEDGMENTS}

This work has been financed by the German Research Foundation (DFG) within project No. DFG-FOR 371, "Peloide." We thank G. Gudehus, G. Huber, M. Külzer, L. Harnau, J. Reinshagen, S. Richter, and M. Bier for valuable collaboration. We thank M. Strauss, A. Komnik, E. Tuzel, D. M. Kroll, A. J. Wagner, Y. Inoue, and M. E. Cates for helpful discussions. T. Ihle thanks the SFB 404, Project No. A7, of the DFG and ND EPSCoR through NSF Grant No. EPS0132289 for financial support.
[1] J. Mahanty and B. W. Ninham, Dispersion Forces (Academic Press, London, 1996).

[2] G. Lagaly, O. Schulz, and R. Zimehl, Dispersionen und Emulsionen (Steinkopff Verlag, Darmstadt, Germany, 1997).

[3] D. J. Shaw, Introduction to Colloid and Surface Chemistry (Butterworth-Heinemann, Oxford, 1992).

[4] I. D. Morrison and S. Ross, Colloidal Dispersions: Suspensions, Emulsions and Foams (John Wiley and Sons, New York, 2002).

[5] K. S. Schmitz, Macroions in Solution and Colloidal Suspension (John Wiley and Sons, New York, 1993).

[6] R. J. Hunter, Foundations of colloid science (Oxford University Press, Oxford, 2001).

[7] S. Richter and G. Huber, Granular Matter 5, 121 (2003).

[8] R. Oberacker, J. Reinshagen, H. von Both, and M. J. Hoffmann, Ceram. Trans. 112, 179 (2001).

[9] G. Wang, P. Sarkar, and P. S. Nicholson, J. Am. Ceram. Soc. 82, 849 (1999)

[10] J. A. Lewis, J. Am. Ceram. Soc. 83, 2341 (2000).

[11] M. Hütter, J. Colloid Interface Sci. 231, 337 (2000).

[12] D. Petera and M. Muthukumar, J. Chem. Phys. 111, 7614 (1999).

[13] P. Ahlrichs, R. Everaers, and B. Dünweg, Phys. Rev. E 64, 040501 (2001).

[14] A. J. C. Ladd and R. Verberg, J. Stat. Phys. 104, 1191 (2001).
[15] R. Adhikari, M. E. Cates, K. Stratford, and A. Wagner, e-print cond-mat/0402598.

[16] O. B. Usta, A. Ladd, and J. Butler, J. Chem. Phys. 122, 094902 (2005).

[17] L. E. Silbert, J. R. Melrose, and R. C. Ball, Phys. Rev. E 56, 7067 (1997).

[18] A. Malevanets and R. Kapral, J. Chem. Phys. 110, 8605 (1999).

[19] A. Malevanets and R. Kapral, J. Chem. Phys. 112, 7260 (2000).

[20] J. T. Padding and A. A. Louis, Phys. Rev. Lett. 93, 220601 (2004).

[21] H. Noguchi and G. Gompper, Phys. Rev. Lett. 93, 258102 (2004).

[22] I. Ali, D. Marenduzzo, and J. Yeomans, J. Chem. Phys. 121, 8635 (2004).

[23] K. Tucci and R. Kapral, J. Chem. Phys. 120, 8262 (2004).

[24] W. B. Russel, D. A. Saville, and W. Schowalter, Colloidal Dispersions (Cambridge University Press, Cambridge, England, 1995).

[25] S. Schwarzer, Phys. Rev. E 52, 6461 (1995).

[26] M. P. Allen and D. J. Tildesley, Computer Simulation of Liquids (Clarendon Press, Oxford, 1987).

[27] Y. Inoue, Y. Chen, and H. Ohashi, J. Stat. Phys. 107, 85 (2002).

[28] M. Ripoll, K. Mussawisade, R. G. Winkler, and G. Gompper, 
Europhys. Lett. 68, 106 (2004).

[29] A. Lamura, G. Gompper, T. Ihle, and D. M. Kroll, Europhys. Lett. 56, 319 (2001).

[30] T. Ihle and D. M. Kroll, Phys. Rev. E 63, 020201(R) (2001).

[31] E. Tuzel, M. Strauss, T. Ihle, and D. M. Kroll, Phys. Rev. E 68, 036701 (2003).

[32] T. Ihle and D. M. Kroll, Phys. Rev. E 67, 066705 (2003).

[33] T. Ihle and D. M. Kroll, Phys. Rev. E 67, 066706 (2003).

[34] T. Ihle, E. Tuzel, and D. M. Kroll, Phys. Rev. E 70, 035701(R) (2004).

[35] N. Kikuchi, C. M. Pooley, J. F. Ryder, and J. M. Yeomans, J. Chem. Phys. 119, 6388 (2003).
[36] E. Falck, J. M. Lahtinen, I. Vattulainen, and T. Ala-Nissila, Eur. Phys. J. E 13, 267 (2004).

[37] R. G. Winkler, K. Mussawisade, M. Ripoll, and G. Gompper, J. Phys.: Condens. Matter 16, S3941 (2004).

[38] R. Blaak, S. Auer, D. Frenkel, and H. Löwen, J. Phys.: Condens. Matter 16, S3873 (2004).

[39] D. M. Heyes, Chem. Phys. 82, 285 (1983).

[40] J. F. Richardson and W. N. Zaki, Trans. Inst. Chem. Eng. 32, 35 (1954).

[41] M. Hütter, Ph.D. thesis, Swiss Federal Institute of Technology Zurich, 1999. 